\title{
SINDICALIZACIÓN EN MÉXICO DURANTE EL PERIODO 1984-2006
}

\section{UNIONIZATION IN MEXICO DURING THE NEOLIBERAL PERIOD 1984-2006}

\section{Roberto Zepeda Martínez*}

RESUMEN

Este artículo analiza las variaciones de la densidad sindical en México durante el periodo 1984-2006. Se examina la afiliación sindical por industria, por ocupación y por región, así como por características socio-demográficas de los miembros sindicales. Se hace una evaluación general de los niveles de la densidad sindical en décadas recientes, de acuerdo a datos oficiales y publicaciones académicas. Los datos demuestran que hubo un declive de la sindicalización en la fuerza laboral total entre 1989 y 1996.

PALABRAS CLAVE: MÉXICO * SINDICALISMO * TRABAJADOR * SINDICATOS * NEOLIBERALISMO

\section{ABSTRACT}

This article analyzes the variations of union density in Mexico during the period 19842006. Union affiliation is examined by industry, occupation and region, as well as by sociodemographic characteristics of union members. A general assessment of unionization levels in recent decades is conducted, according to official data and academic publications. Statistical data, demonstrate a decline of unionization in total labor force between 1989 and 1996.

KEYWORDS: MEXICO * TRADE UNIONISM * WORKER * TRADE UNIONS * NEOLIBERALISM

Instituto de Estudios Internacionales de la Universidad del Mar, México.

zepeda_roberto@yahoo.com

Este articulo se deriva de los resultados del proyecto de investigación "Democratización, Neoliberalismo, y Sindicalismo en Latinoamérica durante el periodo 1980-2010” (CuP-21EI1406) 


\section{INTRODUCCIÓN}

Un drástico declive de las tasas de densidad sindical ocurrió en la mayoría de los países en desarrollo $y$ desarrollados durante las últimas tres décadas. En el caso de México, esto sucedió más claramente en la primera mitad de los 90 , pero la densidad sindical ${ }^{1}$ permaneció estable entre 1996 y 2006. De hecho, la sindicalización tuvo un incremento durante la segunda mitad de los 80 . Por esta razón, este artículo se enfoca en el análisis de la membresía sindical $y$ en las variaciones de la tasa de densidad sindical en México durante el periodo 1984-2006.

En la consecución de este propósito, se examinará la afiliación sindical de acuerdo a las categorías específicas y las características sociodemográficas de los miembros sindicalizados.

En la primera sección, se realizará una evaluación general de la tasa de densidad sindical $y$ sus tendencias en periodos recientes de acuerdo a investigaciones previas en la temática. Las estadísticas analizadas demuestran que las tasas de densidad sindical varían considerablemente de acuerdo a las fuentes consultadas. En la segunda parte, se describen los principales factores que explican el declive de la densidad sindical en México, evaluados de acuerdo a la literatura existente en esta área. En la sección siguiente, la membresía sindical $y$ las tasas de densidad sindical son examinadas basándose en la Encuesta Nacional de Ingresos y Gastos de los Hogares (eNIGH) del Instituto Nacional de Estadística, Geografía e Informática (INEGI). Se analizan las encuestas de los siguientes años: 1984, 1989, 1992, 1994, 1996, 1998, 2000, 2002, 2004 y 2006, como las principales fuentes para calcular los indicadores de sindicalización (cuadro 1). Posteriormente, los datos

1 La densidad sindical se define básicamente como el porcentaje que resulta del número total de trabajadores sindicalizados, respecto de la fuerza laboral total. En México, la densidad sindical se calcula sobre la fuerza laboral activa (Población Económicamente Activa, PEA); no obstante, algunos estudios consideran para el cálculo de la densidad sindical únicamente a la población asalariada dentro del sector formal. En México, existe este dilema debido a que gran parte de la fuerza laboral se encuentra en el sector informal. obtenidos se comparan con las estimaciones hechas por otros autores que usan esta misma fuente. Con base en investigaciones previas, se generará una discusión acerca de los datos generados y presentados en este artículo, en la cual se ofrece una perspectiva de los principales factores que explican el declive de la densidad sindical en México.

\section{DENSIDAD SINDICAL EN MÉXICO: UNA EVALUACIÓN GENERAL}

Los estudios enfocados en la densidad sindical en México presentan diferencias notables, dependiendo de las fuentes y esquemas que se usan para su estimación. Por ejemplo, algunos estudios se basan exclusivamente en la encuesta ENIGH $y$ otros utilizan una combinación de diferentes fuentes oficiales, como la Secretaría de Trabajo y Previsión Social (stPs), InEgr y el Instituto de Seguridad y Servicios Sociales de los Trabajadores del Estado (Issste).

De esta manera, los resultados difieren en gran medida; por ejemplo, los niveles de sindicalización varían desde una tasa del $20 \%$ (Fairris y Levine, 2004) a aproximadamente el 10\% (Aguilar, 2005; Herrera y Melgoza, 2003)2 . De hecho, aparte de estos datos, hay estimaciones del 15\% y del 56\% aparentemente, usando un esquema diferente a los anteriores para calcular la sindicalización (Comisión para la Cooperación Laboral-ccL, 2003 y Zapata, 2005). Además, como se ha destacado por la Organización Internacional de Trabajo- orт (1998), la densidad sindical en México era del 43\% en 1991, pero de acuerdo a la Organización para la Cooperación y el Desarrollo Económico-ocDE (2004) era de $18 \%$ en el año 2000. Estas disparidades no se perciben, por ejemplo, en las estadísticas laborales de países como Estados Unidos, Canadá y el Reino Unido, así como pueden complicar estudios comparativos en diferentes periodos, especialmente por categorías.

$2 \quad$ Por ejemplo, en el año 2000, la membresía sindical en México era de poco más de 4 millones; los trabajadores asalariados del sector formal, alrededor de 20 millones; la PEA, cerca de 40 millones. Si la densidad sindical se calculara sobre los primeros, sería del $20 \%$, pero si se calculara tomando en cuenta a la PEA, sería del $10 \%$. 
Fairris y Levine (2004) sostienen que la densidad sindical en México, se redujo un tercio durante el periodo 1984-2000. Al tomar en cuenta la fuerza laboral en el sector formal, estos autores notaron que la densidad sindical declinó del 30\% en 1984 al 20\% en el año 2000. Ellos afirman que el declive más acentuado ocurrió durante el periodo 1984-1994, con la disminución de 9 puntos porcentuales, quedando en $21 \%$. Posteriormente, esta tasa de densidad sindical se mantendría estable con una fluctuación entre el $22 \%$ y el $20 \%$. Además, la densidad sindical en el país, en relación con la fuerza laboral asalariada del sector formal, ha declinado en un extenso espectro de industrias y ocupaciones (Fairris y Levine, 2004).

Con base en la encuesta ENIGH del INEGI, Panagides y Patrinos (1994) encontraron que la densidad sindical total en México era de alrededor del 30\% en 1989, una estimación que coincide con otros estudios. De igual manera, este estudio reporta que la probabilidad de la membresía sindical es más baja para los hombres que para las mujeres, a diferencia de muchos estudios en naciones desarrolladas, donde las mujeres son menos propensas a ser sindicalizadas que los hombres.

Herrera y Melgoza (2003) indican que la membresía sindical en México era casi del 10\% de la Población Económicamente Activa (PEA) en el año 2000. Un análisis comparativo de las tendencias de la densidad sindical durante el periodo 1992-2000 y desarrollado por estos autores, indica que el porcentaje de trabajadores sindicalizados disminuyó de $13,6 \%$ en 1992 al 9,8\% en el año 2000.

En contraste, los datos presentados por la ccl (2003) revelan que la representación sindical en México, pasó del 23\% en 1985 al 15,6\% en el año 2001. La fuente consultada fue la encuesta enigh; es decir, la misma usada por otros investigadores, pero aparentemente el esquema para calcular la densidad sindical no es similar a aquellos identificados aquí, ya que los resultados finales son ligeramente divergentes. De acuerdo al documento Employment Outlook de la oCDE (2004: 145), la tasa de sindicalización de México era de 43\% en 1990, pero declinó al $18 \%$ en el año 2000 . No obstante, las fuentes de tales estimaciones no son suficientemente de- talladas, por lo que no se puede identificar cuál esquema se usó para el cálculo de la densidad sindical. La OCDE ha publicado estadísticas más recientes sobre sindicalización, en las cuales se puede ver que la densidad sindical de México declinó del 43\% en 1990 al 18\% en el año 2006. Asimismo, en este indicador se percibe una relativa estabilidad en los últimos años.

De acuerdo a la огт (1998), la tasa de sindicalización en México era de 42,8\% en 1991. Este reporte muestra cifras sobre membresía sindical en un amplio grupo de países en el mundo; sin embargo, en el caso de México, solo incluye datos por un año, lo cual no permite hacer estimaciones de las variaciones de la densidad sindical en diferentes años. Como esta tasa de representación sindical es muy similar a la presentada por la ocDE, se considera que el esquema utilizado es prácticamente el mismo.

Cardoso y Gindin (2009) compararon las fluctuaciones de la densidad sindical en Latinoamérica en décadas recientes, en las cuales se evaluaron las tasas de sindicalización en México, Brasil y Argentina. En el primer caso, se observa un declive de la densidad sindical durante el período de 1992-2002 (del 13,6\% al $10 \%)$ pero un ligero aumento de la membresía sindical (de 4,1 a 4,2 millones de miembros). Estos autores hicieron estimaciones de la densidad sindical - basándose en la encuesta ENIGH 2006 - para calcular tasas de sindicalización considerando a los trabajadores asalariados (una fracción de la PEA) para obtener la tasa de sindicalización. De esta manera, la densidad sindical era del 16,5\% en 2006.

Como se ha observado, se ha dado un declive de la densidad sindical total en México. A pesar de que muchos académicos y organizaciones oficiales identificaron una tendencia negativa en décadas recientes, hay una diferencia significativa respecto a los niveles de sindicalización en el país. Lo anterior se debe a que existen diferentes fuentes y esquemas para calcular la densidad sindical.

\section{FACTORES DETERMINANTES DEL DECLIVE DE} LA DENSIDAD SINDICAL

Los factores que explican el declive sindical pueden ser clasificados como cíclicos, 
estructurales y político-institucionales. De acuerdo a varios autores, abogados laborales e instituciones gubernamentales, hay una variedad de factores que determinan la trayectoria de la densidad sindical en México, durante las últimas décadas. La relativa perdida de la membresía sindical ha estado relacionada con factores económicos, estructurales y políticos que ocurrieron desde la década de los 80 .

Fairris y Levine (2004) argumentan que el declive de la densidad sindical en México es atribuible en gran medida, al cambiante contexto estructural-institucional, en el cual los sindicatos involucran nuevos trabajadores y retienen a los miembros existentes. Estos cambios incluyen la modificación de las políticas gubernamentales y la creciente oposición de los empleadores a los sindicatos. Estos autores exponen que solo una pequeña proporción del declive es explicado por cambios en la industria, las ocupaciones y las características socio-demográficas de los trabajadores. En suma, consideran que los factores estructurales y político-institucionales son más relevantes en el deterioro del sindicalismo.

De acuerdo a Bensusán (2004) los principales factores que explican el declive de la densidad sindical en México, están relacionados con factores estructurales y político-institucionales, tales como, la transformación en el mercado laboral y la ineficacia de la expansión de los sindicatos.

Respecto a los factores político-institucionales mencionados previamente, en el caso de México, uno de los factores más relevantes es la existencia de cláusulas de exclusión en los contratos colectivos de trabajo. Por medio de este mecanismo, los trabajadores son forzados a afiliarse al sindicato para obtener un empleo, de lo contrario, serían despedidos. Marshall (2006) identificó un declive de la densidad sindical en México en el periodo 1980-2000, a pesar de la existencia de las cláusulas de exclusión, uno de los instrumentos que les permiten a los sindicatos afiliar trabajadores de manera obligatoria.

Mirón (entrevista, Secretario del trabajo, 2007) identifica dos factores principales que explican el declive sindical en México, especialmente, las políticas neoliberales y el desempeño de las organizaciones laborales. Primero, el declive sindical puede ser atribuido a un factor externo, en esencia, las políticas económicas neoliberales que promueven un modelo económico de libre mercado. Segundo, el declive sindical está relacionado con factores internos, la falta de respuestas efectivas de los sindicatos ante la agenda neoliberal. Evidentemente, la presencia de las estructuras sindicales no son útiles al estado neoliberal, al contrario, dentro de una economía de libre mercado, el gran sector empresarial y las corporaciones transnacionales son apoyadas por el Estado (ibid.).

Cardoso y Gindin, mediante un estudio comparativo sobre las variaciones de la densidad sindical en Argentina, Brasil y México, observan que:

... el paisaje es de retroceso de la sindicalización en México, y de ascenso de la densidad sindical en Argentina y Brasil, con la membresía sindical aumentado entre las mujeres en los tres países, debido principalmente (pero no exclusivamente) a cambios estructurales en el mercado laboral, incluyendo una reducción del empleo en manufactura, $y$ un incremento del empleo en los servicios sociales y el comercio (Traducción propia, 2009: 34).

Algunos analistas laborales en México afirman que ciertos factores políticos e institucionales que regulan las relaciones laborales en el país, han sido más relevantes que las políticas neoliberales para el declive de la membresía sindical y la erosión de la influencia sindical en los ámbitos político y económico. Estos analistas argumentan que las instituciones laborales, tales como, la Secretaría de Trabajo y Previsión Social-stps y las juntas laborales (Junta Local de Conciliación y Arbitraje-JLCA y Junta Federal de Conciliación y Arbitraje-JFCa) obstruyen la organización de sindicatos auténticos e independientes $y$ contribuyen al control ejercido por las compañías sobre los trabajadores; de hecho, sugieren que estas instituciones $y$ organismos laborales son manipulados por el sindicalismo oficial (Arturo Alcalde, entrevista, abogado laboral, 2008; Alfonso Bouzas, entrevista, abogado laboral, 2008 y Néstor De Buen, entrevista, abogado laboral, 2007). 
Desde una perspectiva diferente, Zapata (2005) remarca la relevancia de los sindicatos en México, durante los tiempos neoliberales. A pesar de que se han dado algunas transformaciones en los mercados laborales propiciadas por las políticas neoliberales, la representación de los sindicatos en la burocracia oficial continua siendo importante.

Dan La Botz (2005) enfatiza que la tasa de densidad sindical no representa un buen indicador para medir el poder real de los sindicatos en México, ya que muchos sindicatos están controlados por el gobierno o por los empleadores. Algunos estiman que el $80 \%$ de todos los contratos colectivos en el sector privado son contratos de protección; de esta manera, La Botz destaca que el declive real del poder sindical durante el periodo 1984-2000, fue aún más dramático que lo que las estadísticas sobre sindicalización podrían mostrar.

Por lo tanto, a la luz de las consideraciones previas, es posible identificar una variedad de factores que explican el declive de los sindicatos en México. Aunque existen algunas discrepancias, estas observaciones coinciden en el hecho de que el declive sindical ocurrió desde la década de los 80 y que está relacionado a cambios estructurales en los mercados laborales, a la transición política, a la legislación laboral $y$ al funcionamiento de las instituciones laborales, entre otros factores. El propósito de las siguientes secciones es identificar con mayor detalle el periodo del declive sindical en México, basándose en fuentes oficiales.

\section{ANÁLISIS DE LA MEMBRESÍA SINDICAL EN MÉXICO, 2006}

Se realizó un análisis estadístico para obtener datos relacionados con la membresía sindical en México, tomando como base las encuestas enigh del inegi. Se considera que la ENIGH es la fuente más completa, ya que permite al investigador acceder a una extensa serie de datos estadísticos. También, ofrece un amplio panorama de la membresía sindical y fuerza laboral en diferentes periodos, y en varias categorías.

Los resultados revelan que para 2006, la PEA en el país, incluyendo a los desempleados y al sector informal, era de 44709819 personas, mientras que la membresía sindical representó 4343920 trabajadores. De esta manera, la densidad sindical total en México, con respecto a la PEA, fue de 9,7\%, significativamente más baja que en 1989, cuando fue del 17,8\% (el punto más alto en el periodo) prácticamente el mismo nivel que en 1998 (9,8\%).

Entre 1984 y 2006, la PEA se incrementó un $100 \%$, pero la membresía sindical solo $17 \%$, lo cual generó una pérdida neta de 7 puntos porcentuales en términos de la densidad sindical durante este periodo (cuadro 1). De acuerdo a los datos obtenidos de las encuestas enIGH, durante el periodo 1984-2006, la tasa de densidad sindical total en México declinó del 16,7\% al 9,7\%; posteriormente, este indicador ha permanecido relativamente estable. La sindicalización alcanzó su nivel más alto en 1989 (con el 17,8\%) y el más bajo en el 2006, con el 9,7\%. Por otra parte, la membresía sindical total se incrementó durante este periodo, por ejemplo, en 1984, los miembros sindicales representaban 3,7 millones; esta cifra se elevó a poco más de 4 millones en el año 2000 y continuó esta tendencia hasta llegar a los 4,3 millones en el año 2006.

El incremento más significativo de la membresía sindical ocurrió entre 1984 y 1989. En este periodo, el número de miembros sindicales se incrementó de 3,7 millones a 4,4 millones. De la misma manera, la densidad sindical avanzó del $16,7 \%$ al 17,8\% en este periodo. Por lo tanto, no hubo un declive de la densidad sindical durante gran parte de la década de los 80 , al menos en el periodo de 1984-1989, cuando datos compatibles se pudieron comparar, de acuerdo a cifras obtenidas por las fuentes antes mencionadas.

En contraste, el declive sindical más importante ocurrió entre 1989 y 1996, pasando de una membresía sindical de 4,4 millones a 3,5 millones. Este periodo fue de constante declive, como se puede constatar en el cuadro 1. La densidad sindical también experimentó el declive más acentuado entre 1989 y 1996, al reducirse del 17,8\% al 10,1\%. Como se mencionó anteriormente, las fluctuaciones de la densidad sindical entre 1996 y 2006, han permanecido alrededor del 10\% en el periodo: en 1996, era del $10,1 \%$ y en 2006 , del 9,7\%. 
La densidad sindical fue más alta en las mujeres que en los hombres en el año 2006. La tasa de sindicalización entre las mujeres trabajadoras fue del 10,5\%, mientras que la de los hombres fue del 9,2\%. Estas cifras confirman los niveles de años anteriores, cuando la densidad sindical era más alta entre las mujeres que entre los hombres, en todos los años de la encuesta (1984-2006). Sin embargo, la membresía sindical ha sido más alta en los hombres que en las mujeres, pero esta diferencia se ha reducido en el periodo analizado. Por ejemplo, en 1984, la membresía sindical en las mujeres era 1,1 millones; no obstante, aumentó a 1,8 millones para el año 2006. En contraste, el número de miembros sindicales hombres declinó ligeramente de 2,5 millones en 1984 a 2,4 millones en 2006. De igual manera, más hombres formaban parte de la fuerza laboral que las mujeres. En 1984, la diferencia era más notable, los hombres representaban 16,3 millones, mientras que las mujeres representaban 5,9 millones. Sin embargo, en 2006, el número de hombres que integraban la PEA fue de 27 millones, comparado con los 17,6 millones de las mujeres (cuadro 1). A pesar de que la membresía sindical es mayor en los hombres, las mujeres trabajadoras se sindicalizaron con mayor rapidez que los hombres durante el período.

Los hombres trabajadores registraron un menor aumento en términos de membresía sindical; en 1984, los hombres que eran miembros de un sindicato fueron 2,2 millones; esta cifra aumentó a 2,7 millones en 1992, pero se erosionó a 2,3 millones en el 2000; luego se recuperó en el 2006, alcanzando casi 2,5 millones de miembros.

En suma, mientras que los niveles de membresía sindical entre las mujeres trabajadoras han aumentado significativamente durante el periodo 1992-2006, estos niveles han declinado en el caso de los hombres. En total, la membresía sindical entre las mujeres se incrementó 57\% durante el periodo 1992-2006, mientras que la representación sindical entre los hombres se redujo aproximadamente $9 \%$.

\section{CUADRO 1 \\ MEMBRESÍA SINDICAL Y DENSIDAD SINDICAL EN MÉXICO HOMBRES Y MUJERES. \\ 1984-2006 \\ (EN MILLONES)}

\begin{tabular}{lrrrrrrrrrr}
\hline & 1984 & 1989 & 1992 & 1994 & 1996 & 1998 & 2000 & 2002 & 2004 & 2006 \\
TOTAL & & & & & & & & & & \\
PEA* & 22,312 & 25,222 & 29,583 & 32,827 & 35,261 & 37,891 & 39,299 & 41,505 & 41,045 & 44,709 \\
PO ** & --- & 24,465 & 28,755 & 31,612 & 33,322 & 36,678 & 38,434 & 40,384 & 39,485 & 42,197 \\
Membresía sindical & 3,725 & 4,480 & 4,123 & 3,752 & 3,559 & 3,732 & 4,011 & 4,124 & 4,705 & 4,343 \\
Densidad sindical & $16,7 \%$ & $17,8 \%$ & $13,9 \%$ & $11,4 \%$ & $10,1 \%$ & $9,8 \%$ & $10,2 \%$ & $9,9 \%$ & $11,5 \%$ & $9,7 \%$ \\
HOMBRES & & & & & & & & & & \\
PEA & 16,353 & 18,136 & 20,825 & 22,193 & 22,891 & 24,427 & 25,557 & 26,116 & 25,996 & 27,033 \\
Membresía sindical & 2,566 & 3,187 & 2,766 & 2,253 & 2,100 & 2,268 & 2,328 & 2,426 & 2,794 & 2,494 \\
Densidad sindical & $15,7 \%$ & $17,6 \%$ & $13,3 \%$ & $10,2 \%$ & $9,2 \%$ & $9,3 \%$ & $9,1 \%$ & $9,3 \%$ & $10,7 \%$ & $9,2 \%$ \\
MUJERES & & & & & & & & & & 1,9 \\
PEA & 5,958 & 7,086 & 8,758 & 10,634 & 12,370 & 13,463 & 13,742 & 15,388 & 15,049 & 17,676 \\
Membresía sindical & 1,158 & 1,293 & 1,357 & 1,498 & 1,459 & 1,464 & 1,682 & 1,698 & 1,910 & 1,849 \\
Densidad sindical & $19,4 \%$ & $18,2 \%$ & $15,5 \%$ & $14,1 \%$ & $11,8 \%$ & $10,9 \%$ & $12,2 \%$ & $11,0 \%$ & $12,7 \%$ & $10,5 \%$ \\
\hline
\end{tabular}

*PEA: Población Económicamente Activa.

**PO: Población Ocupada. Datos calculados por el autor con base en cifras de desempleo obtenidas del INEGI, 2011.

Fuente: Elaboración propia a partir de datos expandidos de los micro-datos de la ENIGH. 
MEMBRESÍA SINDICAL POR INDUSTRIA, OCUPACIÓN, ESTADO, EDAD Y EDUCACIÓN

Las tasas más altas de sindicalización por industrias y sectores en 2006, de acuerdo a las clasificaciones de la ENIGH $y$ a estimaciones propias, se dieron en las siguientes categorías: servicios en educación (56\%), electricidad, agua y transmisión de gas (47\%), minería (40\%), servicios de salud $y$ trabajo social (37\%), actividades del gobierno $y$ de organismos internacionales (25\%) e información en medios masivos (24\%).

Por otra parte, con las tasas más bajas de sindicalización se detectaron los siguientes sectores: manufactura (14\%), transporte, correos $y$ almacenamiento (10\%), servicios financieros $y$ de seguros (9\%), servicios de esparcimiento cultural, deportivo y otros servicios recreativos $(6,2 \%)$, servicios de apoyo a los negocios $y$ manejo de desechos y servicios de remediación $(4,5 \%)$, servicios de alojamiento temporal $y$ de preparación de alimentos y bebidas (4\%), comercio al mayoreo y menudeo $(2,3 \%)$ y construcción (1,8\%).

En términos de membresía sindical por industria en 2006, los sectores con más miembros sindicales fueron servicios en educación
(1 264 584), manufactura (1 108 702), actividades del gobierno y de organismos internacionales (480 139), servicios de salud y asistencia social (475 466); los cuales representaron en conjunto alrededor del $75 \%$ de la membresía sindical total agrupada en sectores industriales.

Entre grupos ocupacionales, de acuerdo a las categorías de la eNiGH, las tasas más altas de sindicalización en 2006 se dieron en las siguientes categorías: trabajadores en educación (59\%), operadores de maquinaria fija y equipos en el proceso de fabricación industrial (32\%), jefes de departamento, coordinadores $y$ supervisores en actividades administrativas $y$ de servicios (20\%).

Con respecto a la membresía sindical por ocupación en el 2006, las categorías con más miembros sindicales fueron: trabajadores de la educación (911 367), operadores de maquinaria fija de movimiento continuo $y$ equipos en el proceso de fabricación industrial (547 863), trabajadores de apoyo en actividades administrativas (521 964) y trabajadores en servicios personales en establecimientos (374 259); los cuales representaron en conjunto más del 50\% de los trabajadores sindicalizados en las categorías ocupacionales (cuadro 2 ). 


\section{CUADRO 2 \\ MEMBRESÍA SINDICAL POR OCUPACIÓN EN MÉXICO 2006}

\begin{tabular}{|c|c|c|c|}
\hline OCUPACIONES & $\begin{array}{c}\text { FUERZA } \\
\text { LABORAL } \\
\text { EMPLEADA }\end{array}$ & $\begin{array}{l}\text { MEMBRESÍA } \\
\text { SINDICAL }\end{array}$ & $\begin{array}{l}\text { DENSIDAD } \\
\text { SINDICAL } \\
\text { (PORCENTAJE) }\end{array}$ \\
\hline $\begin{array}{l}\text { Artesanos y trabajadores fabriles en la industria de } \\
\text { la transformación y trabajadores en actividades de } \\
\text { reparación y mantenimiento }\end{array}$ & 6189574 & 291241 & 4,7 \\
\hline $\begin{array}{l}\text { Trabajadores en actividades agrícolas, ganaderas, } \\
\text { silvícolas de caza y pesca }\end{array}$ & 5929440 & 11460 & 0,2 \\
\hline $\begin{array}{l}\text { Comerciantes, empleados de comercio y agentes de } \\
\text { ventas }\end{array}$ & 5924415 & 152982 & 2,6 \\
\hline Trabajadores en servicios personales en establecimientos & 3701463 & 374259 & 10,1 \\
\hline $\begin{array}{l}\text { Ayudantes, peones y similares en el proceso de } \\
\text { fabricación artesanal e industrial y en actividades de } \\
\text { reparación y mantenimiento }\end{array}$ & 3578389 & 180919 & 5,1 \\
\hline $\begin{array}{l}\text { Trabajadores de apoyo en actividades } \\
\text { Administrativas }\end{array}$ & 2794815 & 521964 & 18,7 \\
\hline $\begin{array}{l}\text { Conductores y ayudantes de conductores de maquinaria } \\
\text { móvil y medios de transporte }\end{array}$ & 2228070 & 241684 & 10,8 \\
\hline $\begin{array}{l}\text { Vendedores ambulantes y trabajadores ambulantes en } \\
\text { servicios }\end{array}$ & 1871644 & 4844 & 0,3 \\
\hline Trabajadores en servicios domésticos & 1865915 & 582 & 0,0 \\
\hline $\begin{array}{l}\text { Operadores de maquinaria fija de movimiento continuo } \\
\text { y equipos en el proceso de fabricación industrial }\end{array}$ & 1725276 & 547863 & 31,8 \\
\hline Técnicos & 1619475 & 315376 & 19,5 \\
\hline Trabajadores en educación & 1539120 & 911367 & 59,2 \\
\hline Profesionistas & 1369082 & 185746 & 13,6 \\
\hline $\begin{array}{l}\text { Jefes de departamento, coordinadores y supervisores en } \\
\text { actividades administrativas y de servicios }\end{array}$ & 1158649 & 232889 & 20,1 \\
\hline $\begin{array}{l}\text { Funcionarios y directivos de los sectores públicos, } \\
\text { privado y social }\end{array}$ & 1136961 & 125634 & 11,0 \\
\hline $\begin{array}{l}\text { Trabajadores en servicios de protección y vigilancia y } \\
\text { fuerzas armadas }\end{array}$ & 935388 & 75099 & 8,0 \\
\hline $\begin{array}{l}\text { Jefes supervisores y otros trabajadores de control en la } \\
\text { fabricación artesanal e industrial y en actividades de } \\
\text { reparación y mantenimiento }\end{array}$ & 749799 & 144383 & 19,3 \\
\hline Trabajadores del arte, espectáculos y deportes & 384976 & 25411 & 6,6 \\
\hline Otros & 7368 & 217 & 2,9 \\
\hline TOTAL & 44709819 & 4343920 & 9,72 \\
\hline
\end{tabular}

Fuente: Elaboración propia a partir de estimaciones de la ENIGH, 2006. 
Como se puede observar, las industrias y sectores más sindicalizados son aquellos relacionados con las actividades de educación y del empleo en la manufactura. Estos resultados se pueden explicar debido a dos factores: por una parte, el sindicato más grande no solo en México sino también en Latinoamérica, es el Sindicato Nacional de Trabajadores de la Educación (sNTE), dirigido por Elba Esther Gordillo, entre 1989 y 2013, quien fue la líder sindical más importante en el país en ese periodo, ya que construyó alianzas con líderes políticos, lo que le permitió acceder a recursos para dicho sindicato.

Por otra parte, el alto número de trabajadores sindicalizados en el sector manufacturero se puede atribuir al hecho de que el empleo en las maquiladoras ha aumentado considerablemente durante las últimas décadas. En los cuadros 2 y 3 , se puede observar que una gran parte de la membresía sindical se encuentra en el sector manufacturero; sin embargo, la mayoría de los sindicatos en este sector son sindicatos "fantasma" con contratos laborales de protección dirigidos por organizaciones laborales oficiales (Quintero, 1997 y Bouzas, 2003). Los sindicatos "fantasma" son creados por las empresas en colaboración con los líderes sindicales para impedir que los trabajadores organicen sus propios sindicatos $y$ así defender sus derechos laborales de manera efectiva. Además, los sindicatos "fantasma" tienen contratos laborales de protección para impedir la creación de contratos laborales auténticos.

\section{CUADRO 3 \\ MEMBRESÍA Y DENSIDAD SINDICAL EN MÉXICO POR INDUSTRIA 2006}

\begin{tabular}{|c|c|c|c|}
\hline INDUSTRIA & $\begin{array}{c}\text { FUERZA } \\
\text { LABORAL } \\
\text { EMPLEADA }\end{array}$ & $\begin{array}{l}\text { TRABAJADORES } \\
\text { SINDICALIZADOS }\end{array}$ & $\begin{array}{c}\text { DENSIDAD } \\
\text { SINDICAL } \\
\text { (PORCENTAJE) }\end{array}$ \\
\hline Comercio al mayoreo y menudeo & 8617080 & 194471 & 2,26 \\
\hline $\begin{array}{l}\text { Agricultura, ganadería, aprovechamiento forestal, } \\
\text { pesca y caza }\end{array}$ & 6120027 & 14648 & 0,20 \\
\hline Otros servicios excepto actividades de gobierno & 4009414 & 40925 & 1,00 \\
\hline Construcción & 3935421 & 70054 & 1,80 \\
\hline Industrias manufactureras & 7692879 & 1108702 & 14,41 \\
\hline $\begin{array}{l}\text { Servicios de alojamiento temporal y de preparación de } \\
\text { alimentos y bebidas }\end{array}$ & 3058530 & 113014 & 3,70 \\
\hline Servicios educativos & 2246525 & 1264584 & 56,30 \\
\hline Actividades del gobierno y de organismos internacionales & 1910218 & 480139 & 25,10 \\
\hline Transporte, correos y almacenamiento & 1929081 & 194941 & 10,11 \\
\hline Servicios de salud y asistencia social & 1300317 & 475466 & 36,60 \\
\hline $\begin{array}{l}\text { Servicios de apoyo a los negocios y manejo de desechos y } \\
\text { servicios de remediación }\end{array}$ & 1105626 & 50282 & 4,50 \\
\hline Servicios profesionales científicos y técnicos & 969650 & 15902 & 1,60 \\
\hline $\begin{array}{l}\text { Servicios de esparcimiento, culturales y deportivos y } \\
\text { otros servicios recreativos }\end{array}$ & 411662 & 25324 & 6,20 \\
\hline Servicios financieros $y$ de seguros & 402363 & 37453 & 9,30 \\
\hline Información en medios masivos & 334290 & 80090 & 24,00 \\
\hline Bienes aíces y servicios relacionados & 258646 & 2056 & 0,80 \\
\hline
\end{tabular}




\begin{tabular}{|c|c|c|c|}
\hline INDUSTRIA & $\begin{array}{c}\text { FUERZA } \\
\text { LABORAL } \\
\text { EMPLEADA }\end{array}$ & $\begin{array}{l}\text { TRABAJADORES } \\
\text { SINDICALIZADOS }\end{array}$ & $\begin{array}{c}\text { DENSIDAD } \\
\text { SINDICAL } \\
\text { (PORCENTAJE) }\end{array}$ \\
\hline $\begin{array}{l}\text { Electricidad, agua y suministro de gas por ductos al } \\
\text { consumidor final }\end{array}$ & 234782 & 111815 & 47,60 \\
\hline Minería & 150989 & 60373 & 40,00 \\
\hline Dirección de corporativos y empresas & 9095 & 0 & 0,00 \\
\hline Otros & 13224 & 3681 & 27,84 \\
\hline TOTAL & 44723043 & 4347601 & 9,72 \\
\hline
\end{tabular}

Fuente: Elaboración propia a partir de estimaciones de la ENIGH, 2006.

La mayor concentración de la membresía sindical en México se localiza en un reducido grupo de Estados, en los cuales se concentra la mayor parte de la actividad económica; por ejemplo, en 2006, solo cinco Estados: Distrito Federal (21\%), Estado de México (9,7\%), Nuevo León $(7,5 \%)$, Jalisco $(6,2 \%)$ y Chihuahua $(4,6 \%)$, representaban alrededor de la mitad del total de actividad económica del país.

En el año 2006, el Estado de México era el más sindicalizado del país con 640357 trabajadores sindicalizados, luego el Distrito Federal (366 699), posteriormente, Nuevo León (333 376), el Estado de Veracruz (286 678), Tamaulipas (242 518), Jalisco (240 858) y Coahuila (177 452). Estos seis Estados en conjunto, reúnen alrededor del $40 \%$ de la membresía sindical total en el país. El resto de los Estados no tenía más de 150000 trabajadores sindicalizados, cada uno. Incluso, algunos Estados registraron una membresía sindical menor a 50 000, por ejemplo: Baja California Sur, Zacatecas, Colima y Campeche.

En suma, las regiones del Centro y del Noreste del país concentran la mayoría de las membresías sindicales totales (cuadro 4). En términos de densidad sindical, los Estados con los porcentajes más altos fueron: Tamaulipas (19\%), Nuevo León (17\%), Coahuila (16\%), Quintana Roo (15\%), Baja California Sur (14\%), Tlaxcala (14\%) y Querétaro (13\%). Estos datos sugieren que las tasas de sindicalización más altas no se encuentran en los Estados que concentran la mayor parte de la fuerza laboral, lo que conduce a una baja densidad sindical a nivel nacional. Se observa que los Estados del Noreste, Norte y Centro registran las tasas de sindicalización más altas en el país (cuadro 4). 


\section{CUADRO 4}

MEMBRESÍA SINDICAL EN MÉXICO POR ESTADO 2006

\begin{tabular}{|c|c|c|c|}
\hline ESTADO & $\begin{array}{c}\text { POBLACIÓN } \\
\text { ECONÓMICAMENTE ACTIVA } \\
(\text { PEA) }\end{array}$ & MEMBRESÍA SINDICAL & $\begin{array}{l}\text { DENSIDAD SINDICAL } \\
\text { (PORCENTAJE) }\end{array}$ \\
\hline México & 5849798 & 640357 & 10,95 \\
\hline Distrito Federal (DF) & 3988398 & 366699 & 9,19 \\
\hline Nuevo León & 1913061 & 333376 & 17,43 \\
\hline Veracruz & 3203461 & 286678 & 8,95 \\
\hline Tamaulipas & 1246716 & 242518 & 19,45 \\
\hline Jalisco & 2923048 & 240858 & 8,24 \\
\hline Coahuila & 1083751 & 177452 & 16,37 \\
\hline Puebla & 2530798 & 176121 & 6,96 \\
\hline Guerrero & 1326918 & 129399 & 9,75 \\
\hline Chiapas & 1841552 & 123122 & 6,69 \\
\hline S. Luis Potosí & 1027817 & 123070 & 11,97 \\
\hline Guanajuato & 1940947 & 121923 & 6,28 \\
\hline Chihuahua & 1365555 & 120805 & 8,85 \\
\hline Michoacán & 1638731 & 108148 & 6,60 \\
\hline Sonora & 1021002 & 101494 & 9,94 \\
\hline Querétaro & 704640 & 93685 & 13,30 \\
\hline Sinaloa & 1125572 & 86450 & 7,68 \\
\hline Quintana Roo & 550758 & 85317 & 15,49 \\
\hline Hidalgo & 945975 & 83517 & 8,83 \\
\hline Oaxaca & 1489305 & 80015 & 5,37 \\
\hline B. California & 1235173 & 72947 & 5,91 \\
\hline Durango & 555402 & 69836 & 12,57 \\
\hline Tabasco & 913240 & 67713 & 7,41 \\
\hline Tlaxcala & 450608 & 61248 & 13,59 \\
\hline Morelos & 654391 & 53760 & 8,22 \\
\hline Aguascalientes & 469392 & 53107 & 11,31 \\
\hline Nayarit & 433696 & 52598 & 12,13 \\
\hline Yucatán & 835663 & 50483 & 6,04 \\
\hline Zacatecas & 567477 & 49804 & 8,78 \\
\hline Campeche & 394619 & 28798 & 7,30 \\
\hline B. California Sur & 226286 & 31350 & 13,85 \\
\hline Colima & 256069 & 31272 & 12,21 \\
\hline TOTAL & 44709819 & 4343920 & 9,72 \\
\hline
\end{tabular}

Fuente: Elaboración propia a partir de estimaciones de la ENIGH, 2006. 
La densidad sindical por edad en México en el año 2006, fue más alta entre los trabajadores cuyas edades fluctuaban entre los 36 y 45 años de edad (14\%) y los de 46 a 55 años (13,9\%); asimismo, el grupo de 56 a 65 años registró un $10 \%$ de sindicalización. Por otra parte, las tasas más bajas de sindicalización se dieron en los grupos de 16 a 25 años (6\%) y de 26 a 35 años (10\%). Estas cifras se asemejan a las de otros países como Estados Unidos, Canadá, Reino Unido y otros países desarrollados, donde las tasas de sindicalización son más altas en los trabajadores que tienen entre 30 y 50 años; de igual manera, las tasas de sindicalización más bajas se encuentran en los trabajadores más jóvenes, principalmente en aquellos que tienen entre 18 y 30 años de edad y en los mayores de 50 años (gráfico 1).

GRÁFICO 1

MEMBRESÍA SINDICAL EN MÉXICO POR EDAD 2006

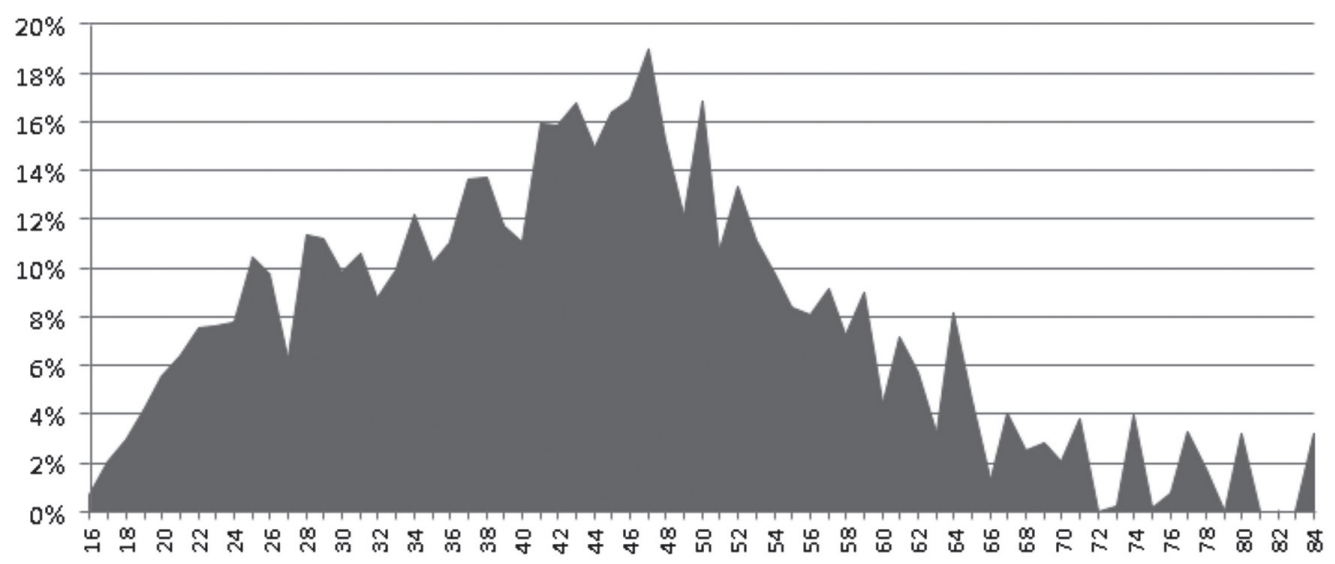

Fuente: Elaboración propia a partir de estimaciones de la ENIGH, 2006.

Estos datos sugieren la falta de interés mostrada por los trabajadores jóvenes para afiliarse a sindicatos y el fracaso de los líderes sindicales en atraer $y$ afiliar a nuevos miembros. De igual manera se podría sugerir que los nuevos empleos están siendo creados en sectores poco propensos para la sindicalización, por ejemplo, en el sector servicios. Al respecto, cabe resaltar que "los jóvenes tienen alta rotación laboral; por el contrario, los individuos de mayor edad, buscan la protección de los sindicatos en lugar de cambiar de ocupación" (McConnell, Brue y Macpherson, 2003: 212-213). Además, estos datos revelan la existencia de trabajo infantil en el país: en el año 2006, alrededor de 1400 trabajadores sindicalizados eran menores de 16 años.
Con respecto a los diferentes niveles $y$ tipo de educación obtenidos por los trabajadores sindicalizados, las tasas de densidad sindical fueron más altas entre aquellos trabajadores con un nivel educativo de licenciatura en escuela Normal ${ }^{3}$ (72\%), doctorado $(40,8 \%)$, maestría $(26,2 \%)$, carrera técnica o comercial $(18,6 \%)$ y profesional $(17,6 \%)$. Por otra parte, con los niveles más bajos de densidad sindical se encuentran los trabajadores con los siguientes niveles de educación: preparatoria $(10,8 \%)$, secundaria $(9,0 \%)$, pre-escolar $(4,2 \%)$ y primaria $(3,3 \%)$. Finalmente, la categoría de trabajadores con ningún tipo de educación registró el 1,0\%.

3 Las escuelas normales son las encargadas de la formación de los maestros de Educación Básica. 
Sin embargo, en términos de la distribución de la membresía sindical, las categorías de trabajadores que tuvieron mayor número de sindicalizados fueron (en orden de importancia): profesional, secundaria, preparatoria, primaria, carrera técnica o comercial, normal o Licenciatura en enseñanza y educación. Se detectó que los grupos más sindicalizados estaban entre aquellos trabajadores con niveles de educación profesional y de secundaria (cuadro 5). Los altos niveles de educación que posee gran parte de los miembros sindicalizados en el país se podrían alcanzar, debido a que la mayoría de trabajadores en el sector de educación son graduados de universidades, mientras que es altamente factible que los trabajadores en las maquiladoras o en la industria manufacturera, tengan la educación secundaria como el nivel más alto.

\begin{tabular}{|c|c|c|c|}
\hline \multicolumn{4}{|c|}{$\begin{array}{c}\text { CUADRO } 5 \\
\text { MEMBRESÍA SINDICAL POR NIVEL DE EDUCACIÓN } \\
2006\end{array}$} \\
\hline NIVEL EDUCATIVO & PEA & MEMBRESÍA SINDICAL & $\begin{array}{l}\text { DENSIDAD SINDICAL } \\
\text { (PORCENTAJE) }\end{array}$ \\
\hline Profesional & 6066630 & 1065329 & 17,60 \\
\hline Secundaria & 11840475 & 1062873 & 9,00 \\
\hline Preparatoria & 6281077 & 678915 & 10,80 \\
\hline Primaria & 13997799 & 465752 & 3,30 \\
\hline Carrera Técnica o Comercial & 2483089 & 462535 & 18,60 \\
\hline Normal & 540662 & 389012 & 72,00 \\
\hline Maestría & 609694 & 159467 & 26,20 \\
\hline Doctorado & 78591 & 32039 & 40,80 \\
\hline Ninguno & 2775929 & 26495 & 1,00 \\
\hline Pre-escolar & 35873 & 1503 & 4,20 \\
\hline TOTAL & 44709819 & 4343920 & 9,70 \\
\hline
\end{tabular}

Fuente: Elaboración propia a partir de estimaciones de la ENIGH, 2006.

ANÁLISIS COMPARATIVO DE LA DENSIDAD SINDICAL: 1984, 1998, 2006

Se puede constatar que las tasas de densidad sindical en México declinaron entre 1984 y 2006, en la mayoría de los sectores e industrias. El declive más drástico ocurrió en el sector de transporte, comunicaciones, correo y almacenaje, el cual pasó de 50,3\% en 1984 al
$12 \%$ en 2006 . Luego, en el sector de minería, electricidad, agua y gas, el cual tenía una densidad sindical de $63,9 \%$ pero se redujo al $45 \%$. De igual manera, el declive sindical aconteció en el sector manufacturero, del $29 \%$ al $14 \%$. También, los servicios sufrieron una caída en la sindicalización, del 37\% en 1984 al 15\% en 2006 (gráfico 2). 
GRÁFICO 2

DENSIDAD SINDICAL POR INDUSTRIA EN MÉXICO

1984-2006

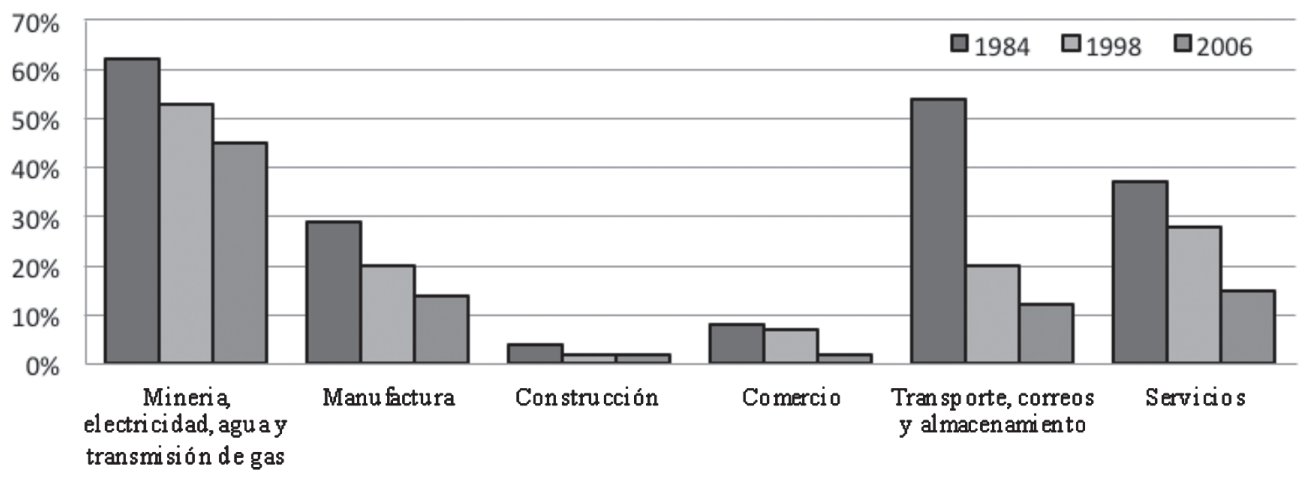

Fuente: Elaboración a partir de Fairris y Levine, 2004 para los años 1984 y 1998, y de la ENIGH para el 2006.

Entre los grupos ocupacionales, las tendencias ilustran una reducción de los niveles de la densidad sindical en la mayoría de las categorías, por ejemplo, la sindicalización en los trabajadores de la educación era del 73\% en 1984 , pero disminuyó al 59\% en 2006. Otra categoría fue la de técnicos: $47 \%$ de estos trabajadores estaban sindicalizados en 1984, pero este porcentaje se redujo al 19\% en 2006. De la misma manera, los trabajadores del arte, espectáculos $y$ deportes sufrieron una reducción en la sindicalización: del 73\% al 7\%. La única categoría que presentó un incremento de la densidad sindical fue en los operadores de maquinaria fija de movimiento continuo $y$ equipos en el proce- so de fabricación industrial, en la cual la densidad sindical estuvo fluctuando entre el 30\% y el 20\% (1984 y 1998), pero después alcanzó el $32 \%$ (2006).

El resto de las categorías ocupacionales mostró una disminución de la representación sindical. Los trabajadores de la categoría de servicios de protección y vigilancia y fuerzas armadas presenciaron un declive persistente: en 1984, la sindicalización era del 27\% y en 2006 bajó al 8\%. Por otra parte, la tasa de sindicalización en los trabajadores profesionistas tuvo un aumento del $13 \%$ al $21 \%$ en el periodo 1984-1998, pero luego, decreció al 14\% (gráfico 3). 


\section{GRÁFICO 3 \\ DENSIDAD SINDICAL EN MÉXICO POR OCUPACIÓN \\ 1984-2006}

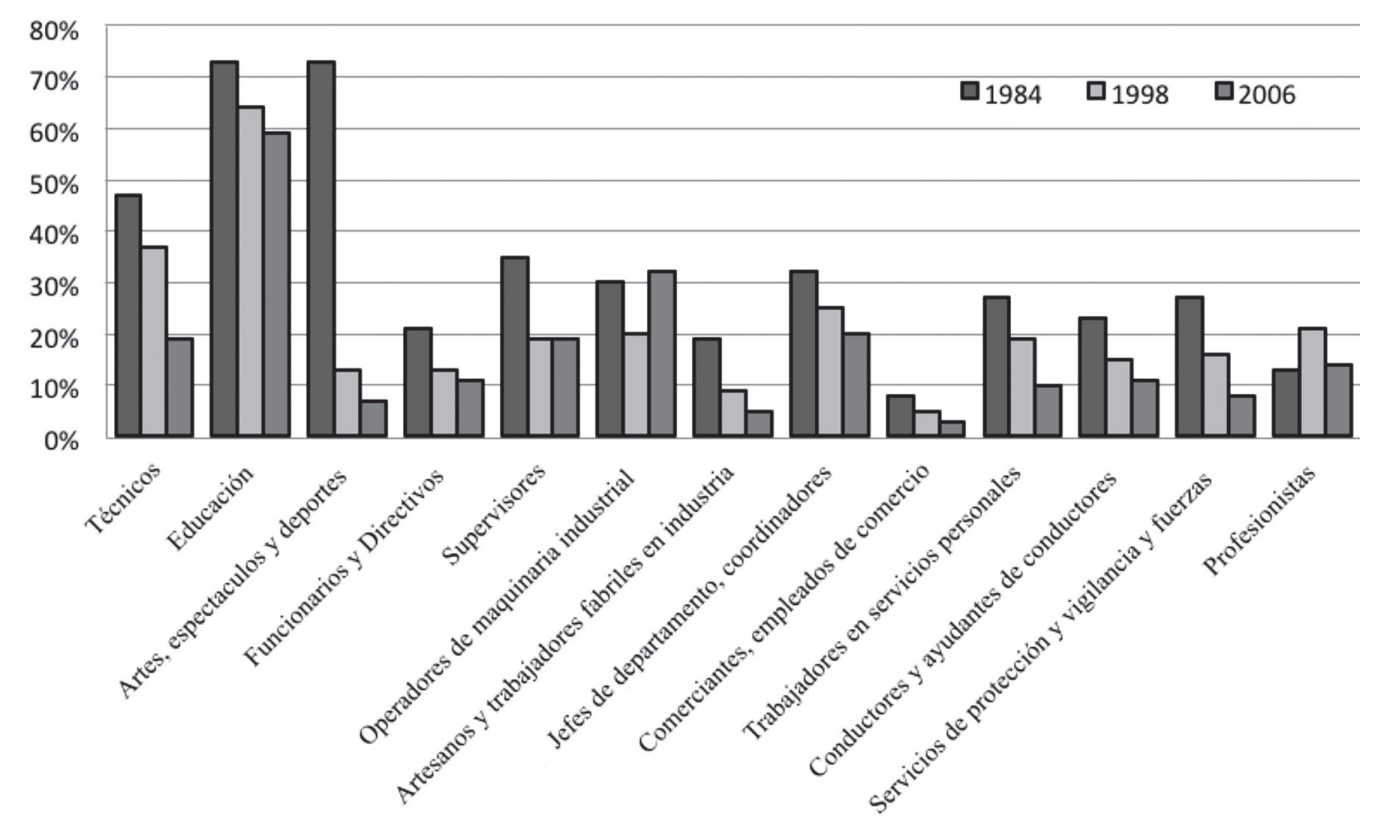

Fuente: Elaboración a partir de Fairris y Levine, 2004 para los años 1984 y 1998, y de la ENIGH para el 2006.

La densidad sindical por grupos de edades también se vio disminuida durante el periodo 1984-2006. La sindicalización entre los trabajadores cuya edad fluctúa entre los 36 y 45 años pasó del 21\% al 16\% (1984-2000), y luego disminuyó al 14\% en el 2006. De igual manera, la densidad sindical cayó entre los trabajadores con edades entre los 26 y 35 años, del 16\% al $10 \%$ en el periodo 1984-2006. El declive también ocurrió en el grupo de trabajadores con edades entre 16 y 25 años, del 12\% al 6\% (19841998), pero permaneció estable en ese nivel hasta el 2006. La categoría de trabajadores de
46 a 55 años se redujo del 18\% al 14\% en el periodo 1984-2006 (gráfico 4). Un aspecto a destacar en estos datos, es la existencia de trabajo infantil en México: los trabajadores de 15 años o menos representaron 1133203 en 2006, de los cuales 1404 menores estaban sindicalizados. Estas cifras confirman los reportes de diversos periódicos acerca del trabajo de niños y adolescentes, principalmente en el sector agrícola. Los Estados que concentraron un mayor número de trabajo infantil en 2006, fueron Veracruz (111 899), Chiapas (98 444), Jalisco (86 133) y Puebla (68 884). 


\section{GRÁFICO 4 \\ DENSIDAD SINDICAL POR EDAD EN MÉXICO \\ 1984, 1996, 2006}

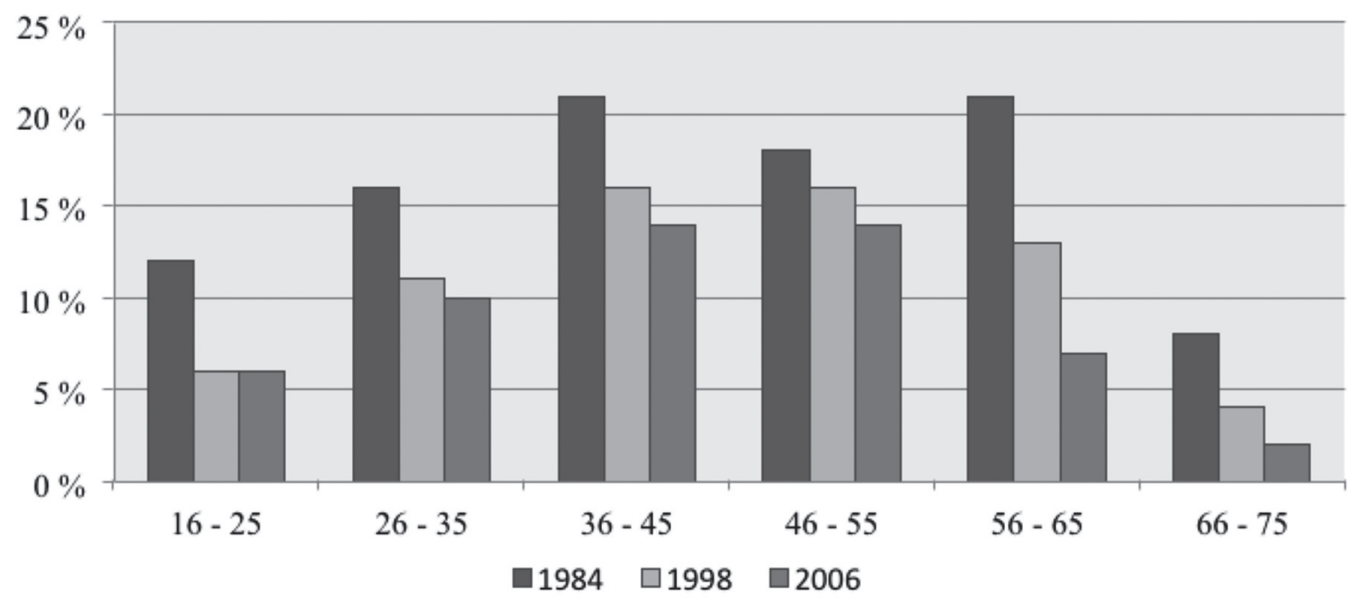

Fuente: Elaboración a partir de Fairris y Levine, 2004 para los años 1984 y 1998, y de la ENIGH para el 2006.

Como se ha demostrado por medio de los datos examinados, ha acontecido un evidente declive de las tasas de densidad sindical en la mayoría de las industrias, sectores y ocupaciones en México, durante el periodo 1984-2006; las excepciones a este tendencia representan una minoría. Conviene observar los factores que propiciaron estas tendencias e identificaron el periodo de mayor declive sindical $y$ de esa manera, descubrir los factores que podrían explicar la caída de las tasas de sindicalización en México.

\section{ANÁLISIS DEL DECLIVE DE LA DENSIDAD SINDICAL EN MÉXICO}

El declive de los sindicatos puede ser analizado desde varias perspectivas, por ejemplo, considerando factores político-institucionales, el desempeño económico, el desempleo (factores cíclicos) y la composición del empleo (factores estructurales). Las repercusiones del ciclo económico y las características de los mercados laborales son pertinentes para explicar el declive de la densidad sindical. En el caso de México, factores como el desempeño económico y las características de la composición del empleo, prevalecientes durante las últimas tres décadas, no han sido adecuados para fomentar una expansión de la densidad sindical. El desempeño económico ha sido pobre y en algunos periodos ha sido negativo; la creación de empleos en el sector formal no ha sido suficiente para emplear a la fuerza laboral disponible en el país, conduciendo a la informalidad de los mercados laborales y complicando la organización de nuevos miembros sindicales.

Respecto a los factores político-institucionales, es pertinente destacar que las interrelaciones entre los sindicatos, los empleadores $y$ el Estado fueron reguladas por una estructura corporativa establecida durante la administración del presidente Lázaro Cárdenas (19341940). En este contexto, desde finales de los 30 hasta principios de los 80 , los sindicatos se convirtieron en actores relevantes dentro del sistema político como un sector influyente en el partido hegemónico (PRI). La estructura corporativa fue diseñada por el Estado con el propósito de promover el proceso de industrialización en México después de la segunda posguerra, ya que se requería de un movimiento laboral manejable en términos de su posición hacia el capital y hacia el Estado. 
La relación del movimiento laboral organizado hacia el Estado contribuyó a la relativa estabilidad de México durante ese periodo. Esta situación facilitó la creación de varias instituciones destinadas al mejoramiento $y$ bienestar de la clase trabajadora, con beneficios sociales tales como cuidado de la salud, pensiones $y$ vivienda, entre otros. No obstante, estas prestaciones se modificaron con el advenimiento del neoliberalismo y la democratización política en las dos últimas décadas del siglo xx; dichos procesos minaron la posición que los sindicatos tenían durante el periodo de posguerra.

Desde la década de los 80 , los gobiernos tecnócratas en México retiraron el apoyo político a las confederaciones y sindicatos oficiales. Tales gobiernos promovieron, en la práctica, una estructura laboral más flexible a las condiciones impuestas por la globalización neoliberal adoptada desde 1982. En estas circunstancias, los sindicatos iniciaron un proceso de deterioro.

Los factores estructurales también fueron importantes. Hacia 2006, la gran franja de sindicalización en México estaba localizada en los sectores público, educativo y de manufactura. Los datos analizados confirman que estos segmentos de la fuerza laboral empleada son los más sindicalizados en el país. Esta situación puede ser atribuida a la legislación laboral, la cual establece que todos los empleados del sector público tienen que estar sindicalizados de manera obligatoria. En la misma lógica, los trabajadores de la educación tienen el sindicato más grande en el país (SNTE); en este sector, la sindicalización es requisito para obtener o mantener el empleo. Los altos niveles de sindicalización en el sector manufacturero se deben principalmente a la expansión de las maquiladoras en el periodo de estudio; sin embargo, en otros sectores e industrias, la representación sindical es baja en el país, lo cual refleja tanto una caída en las tasas de sindicalización como en la capacidad de los sindicatos por mantener y expandir su membresía.

El declive de la densidad sindical también está relacionado con el estancamiento del empleo formal en el sector privado, especialmente en la primera mitad de la década de los 90. Entre 1990 y 1995, el número de empleos en el sector privado formal aumentó ligeramente de 9,3 millones a 9,4 millones (es decir, menos de 100000 en 5 años). Esto podría ser considerado como un factor que afectó la afiliación sindical de los trabajadores en este periodo. También es destacable que en el periodo 1989. 1994, caracterizado por relativamente altas tasas de crecimiento económico, a diferencia de la década de los 80, no estuvo acompañada por la expansión de empleos formales en el sector privado.

De esta manera, los factores estructurales jugaron un rol importante en el declive de la densidad sindical. Además, la crisis del peso de 1994-1995, generó efectos que fueron negativos para la afiliación sindical, por ejemplo, la caída del empleo y la expansión del sector informal. Lo anterior puede ser corroborado con el análisis de la fuerza laboral empleada $y$ otros indicadores, de acuerdo a estadísticas oficiales del gobierno de México.

A partir del 2000, había un movimiento sindical reducido en comparación con décadas previas, desde varias perspectivas: en términos de su influencia en la formulación de políticas públicas favorables para los trabajadores y sindicatos, en términos de su relación y alianza con los partidos políticos y el Estado, $y$ finalmente, en términos de su representación dentro de la fuerza laboral empleada. Además, la disminución del movimiento sindical en México impidió una estrategia efectiva para negociar con el Estado, la atenuación de las repercusiones de la transición dual, en aspectos tales como, la privatización y la reducción de recursos públicos, así como las políticas sociales y económicas que impactaban en la clase trabajadora; sin embargo, al mismo tiempo, los sindicatos oficiales demostraron influencia en el entorno político previniendo la reforma laboral. Sindicatos oficiales afiliados a la CTM representan a la mayoría de los trabajadores sindicalizados en el sector manufacturero $y$ en las industrias petrolera y eléctrica. Otras organizaciones independientes, como la UNT, juegan un rol importante en el movimiento laboral y representan a los trabajadores en el sector de telecomunicaciones, en el cuidado de la salud y en el sector universitario. 


\section{CONCLUSIONES}

Como ha sido el caso de otros países desarrollados, en México también se presentó un declive de la densidad sindical en las últimas décadas. El análisis de estudios previos, así como las estadísticas generadas $y$ analizadas en esta investigación, revelan un declive de la densidad sindical total y desagregada en la gran mayoría de sectores, ocupaciones y considerando algunas características socio-demográficas de los miembros sindicalizados.

El análisis de la densidad sindical demuestra un declive más notable entre 1989 y 1996. Esto sugiere que ciertas políticas públicas, implementadas en el país en ese periodo, propiciaron una reducción de los trabajadores sindicalizados en la fuerza laboral. Por una parte, el conjunto de políticas neoliberales aplicadas durante la administración del presidente Carlos Salinas (1988-1994); por otra parte, la crisis económica de los años 19941995, generó fuertes repercusiones para la clase trabajadora, ya que tanto el desempleo como el empleo informal aumentaron. Estos factores, en un contexto político autoritario, resultaron aún más perniciosos para la afiliación de nuevos miembros sindicales y el mantenimiento de los miembros existentes. El aumento del sector informal, alrededor del $50 \%-60 \%$ de la fuerza laboral, ha complicado el panorama para los sindicatos, ya que la expansión de la membresía sindical es más difícil en este escenario.

\section{BIBLIOGRAFÍA}

\section{LIBROS}

Aguilar, Javier. La población trabajadora y sindicalizada en México en el período de la globalización. México: Facultad de Ciencias Económicas de la Universidad Nacional Autónoma de México (FCEUNAM), 2005.

Bensusán, Graciela. "A new scenario for Mexican trade unions: changes in the structure of political and economic opportunities". Dilemmas of political change in Mexico. Kevin Middlebrook (ed.). North Carolina, EEuU: Institute for the Study of the America, 2004.

Bouzas, Alfonso. Reforma laboral; análisis crítico del Proyecto Abascal de reforma a la Ley Federal de Trabajo. México: Universidad Autónoma de México-unAm, 2003.

Comisión para la Cooperación Laboral-CCL. Recent trends in union density in North America. Washington, EEUu: CCL, 2003.

De la Garza, Enrique. "La crisis de los modelos sindicales en México y sus opciones". La situación del trabajo en México. Enrique De la Garza y Carlos Salas (eds.). México: Plaza y Valdez, 2003.

Esquinca, Marco T. y Melgoza, Javier. "La afiliación sindical y premio salarial en México". La situación del trabajo en México. Enrique De la Garza y Carlos Salas (eds.). México: Plaza y Valdez, 2003.

McConnell, Campbell; Brue, Stanley y Macpherson, David. Economía laboral. España: McGraw Hill, 2003

Organización Internacional del Trabajo-oIT. World Labor Report 1997-1998. Ginebra, Suiza: OIT, 1998.

Organización para la Cooperación y el Desarrollo Económico-ocde. Employment outlook. Paris, Francia: OCDE, 2004.

Panagides, Alexis y Patrinos, Harry. "Unionnonunion wage differentials in the developing world: a case study of Mexico". Policy, Research Working Paper. Washington DC, EEuU: World Bank, 1994. En: <http://documents.worldbank. org/curated/en/1994/03/698534/ unionnonunion-wage-differentials-developingworld-case-study-mexico> [consultado el 10 de julio de 2009].

Quintero, Cirila. Reestructuración sindical en las maquiladoras mexicanas. México: El Colegio de la Frontera Norte-colef, 1997.

Zapata, Francisco. Tiempos neoliberales en México. México: El Colegio de México, 2005.

\section{PUBLICACIONES PERIÓDICAS}

Cantú, Jesús. "Los brazos del SNTE". Revista Proceso 1719. México, Comunicación 
e Información sA, 2009. [Revista digital]. En <http://www.proceso.com. $\mathrm{mx} / \mathrm{p}=119397>$ [consultado el $12 \mathrm{de}$ octubre de 2009].

Ebbinghaus, Bernard. "Globalization and trade unions: a comparative-historical examination of the convergence thesis". Economie Appliquée: An International Journal of Economic Analysis 55. Paris, Francia. Institut de Sciences Mathématiques et Economiques Appliquées-ISMEA, 2002: 121-139.

Fairris, David y Levine, Edward. "Declining union density in Mexico, 1984-2000". Monthly Labor Review 127 (9). EEUU, Us. Department of Labor, 2004: 10-17.

Herrera, Fernando y Melgoza, Javier. "Evolución reciente de la afiliación sindical y la regulación laboral en México". La situación del trabajo en México. Enrique De la Garza y Carlos Salas (eds.). México. Plaza y Valdez, 2003: 323-347.

La Botz, Dan. "Mexico's labor movement in transition". Monthly Review 57 (2). EEUU. Monthly Review Foundation, 2005: 62-72.

\section{TEXTOS ELECTRÓNICOS}

Cardoso, Adalberto y Gindin, Julián. "Industrial relations and collective bargaining: Argentina, Brazil and Mexico compared". Working Paper 5. Ginebra, Suiza: Industrial and Employment Relations Department International Labour Office, 2009. En: <http://www.ilo.org/ wcmsp5/groups/public/---ed_dialogue/--dialogue/documents/publica tion/ wcms_158020.pdf> [consultado el 15 de octubre de 2010].
OTROS

Instituto Nacional de Estadística, Geografía e Informática-INEGI. Encuesta Nacional de Ingresos y Gastos de los Hogares (ENIGH) 2006. México: INEGI, 2007.

Instituto Nacional de Estadística, Geografía e Informática-INEGI. Micro-datos de la Encuesta Nacional de Ingresos y Gastos de los Hogares-ENIGH). México, 1984, 1989, 1992, 1994, 1996, 1998, 2000, 2002, 2004 y 2006. En <http://www. inegi.gob.mx $>$ [consultado el $1^{\circ}$ de julio de 2009].

Marshall, Adriana. Efectos de la regulación del trabajo sobre la afiliación sindical: estudio comparativo de Argentina, Chile y México. Argentina: Cuadernos del Instituto de Desarrollo Económico y Social, 2006.

Entrevistas

Arturo Alcalde. Abogado laboral y asesor de sindicatos (Frente Auténtico del TrabajoFAT, Unión Nacional de Trabajadores-UnT, entre otros). México Distrito Federal. Setiembre, 2007.

Alfonso Bouzas. Abogado laboral e investigador académico. Universidad Nacional Autónoma de México-unam. México Distrito Federal. Setiembre, 2007.

Néstor De Buen. Abogado laboral y asesor sindical (Sindicato Nacional de Trabajadores Mineros y MetalúrgicosSNTMm y otros). México Distrito Federal. Setiembre, 2007.

Benito Mirón. Secretario del Trabajo. Gobierno de Marcelo Ebrard. México Distrito Federal. Noviembre, 2007.

Fecha de ingreso: 09/09/2013 Fecha de aprobación: 31/01/2014 
